\title{
A Software Framework for Individualized Agent Behavior
}

\author{
Ionut Damian, Birgit Endrass, Nikolaus Bee, and Elisabeth André \\ Human Centered Multimedia, Institute of Computer Science, \\ Augsburg University, 86159 Augsburg, Germany
}

\section{Introduction and Motivation}

Inter-agent interactions play an important part in virtual simulations of social behavior. Such interactions help visualize and analyze theoretical findings and assumptions in key sociological domains. However, most computer applications that simulate agent behavior lack the flexibility and adaptability researchers need to visualize theoretical concepts. This shortcoming can also be observed in modern computer games. For example, while most online games allow the player to customize his or her character, this customization is only aesthetical, such as hair color or outfit. These games do not allow the customization of the way the player's character behaves, for example how it gazes, how fast it gesticulates or what position it will take during an interaction. Strong and intelligent parametrization of the virtual agents is a solution to this problem. Each agent can have its own set of preferred gazing techniques, positions during an interaction and execute gestures in a specific way. Placing these custom agents together in a scene would then create a realistic and diverse virtual environment.

\section{The System}

An implementation of the above mentioned approach represents the Advanced Agent Animation (AAA) framework ${ }^{1}$. This framework is able to reproduce complex yet customizable agent interactions, using a powerful action parametrization system covering all important aspects of an agent interaction: body location, body orientation, gestures, gazing and movement. The generation of the low-level behavior of agents is automated and the framework enables the user or external systems to control the high-level behavior.

Formation System. To represent inter-agent interactions, the system uses Fformations [2]. Agents can join together to form formations. While in a formation, an agent will always try to satisfy its preferences for interpersonal distance and orientation. Possible conflicts between agents' interpersonal distance constraints are explicitly handled by the system.

The framework automatically generates the low-level behavior of positioning and orienting agents during an interaction. This can be customized by altering

${ }^{1}$ http://hcm-lab.de/projects/aaa/ 
an agent's parameters for interpersonal distance, willpower, deviation from the default orientation, and preferred formation type.

Animation System. The animation management system is responsible for the loading, management and playback of animations. The system is capable of dealing with indeterministic animation playback requests. For this it employs a powerful animation blending system which is able to realistically start the playback of an animation even if other animations are already being rendered on the agent. The expressivity [1] of each animation can be customized with the help of the parameters: fluidity, stroke repetitions, playback speed and spatial extent.

Movement System. Agents can be placed inside a virtual scene and are able to locomote through the scene and orient themselves to face specific objects or coordinates. Each time an agent moves from one point in the scene to another, a movement action is performed. The movement actions can be customized with the help of the parameters: walking speed and movement animation.

Gazing System. The virtual agents are able to move their head and eyes to gaze towards specific targets, may these be points in space, objects or other agents. The system automatically generates gazing actions for an agent when it shows interest in another agent's action. The user or external systems can also request gazing actions. These can be customized with the help of the parameters: morphing speed and gaze duration.

\section{Conclusion}

This paper presented an approach to individualizing interactions between virtual agents by describing a software framework for generating non-verbal behavior of virtual agents. The major strength of the framework is the high customizability of the behavior generation processes. It is plausible that such a feature can support user immersion in video games by giving users the opportunity to create avatars with which they can identify themselves more easily.

Acknowledgments. This work has been funded in part by the European Commission under the grant agreement DynaLearn (FP7-ICT-231526).

\section{References}

1. Hartmann, B., Mancini, M., Pelachaud, C.: Implementing expressive gesture synthesis for embodied conversational agents. Science 3881, 188-199 (2005)

2. Kendon, A.: Conducting Interaction: Patterns of behavior in focused encounters. Cambridge University Press, Cambridge (1990) 